\title{
RESEARCH
}

\section{Glucagon modulates proliferation and differentiation of human adipose precursors}

\author{
Giulia Cantini', Martina Trabucco', Alessandra Di Franco', Edoardo Mannucci1,2 and Michaela Luconi1,2 \\ ${ }^{1}$ Endocrinology Unit, Department of Experimental and Clinical Biomedical Sciences, University of Florence, Florence, Italy \\ 2Azienda Ospedaliera Universitaria Careggi (AOUC), Careggi Hospital, Florence, Italy
}

Correspondence should be addressed to G Cantini or M Luconi: giulia.cantini@unifi.it or michaela.luconi@unifi.it

\begin{abstract}
Glucagon-like peptide 1 receptor agonists (GLP-1RAs), which are currently used for the treatment of type 2 diabetes, have recently been proposed as anti-obesity drugs, due to their relevant effects on weight loss. Furthermore, dual agonists for both GLP-1R and glucagon receptor (GCGR) are under investigation for their promising action on adiposity, although underlying mechanisms still need to be clarified. We have recently demonstrated that GLP-1 and liraglutide interfere with the proliferation and differentiation of human adipose precursors, supporting the hypothesis of a peripheral action of GLP-1RA on weight. Here, we investigated glucagon activity in an in vitro model of primary human adipose-derived stem cells (ASCs). Glucagon significantly inhibited ASC proliferation in a dose- and time-dependent manner, as evaluated by cell count and thymidine incorporation. When added during in vitro-induced adipogenesis, glucagon significantly reduced adipocyte differentiation, as demonstrated by the evaluation of intracellular fat content and quantitative expression of early and mature adipocyte markers (PPAR $\gamma$ and FABP4, HSL). Notably, the inhibitory effect of glucagon on cell proliferation and adipogenesis was reversed by specific GLP-1R (exendin-9) and GCGR (des-His ${ }^{1}$-Glu9'-glucagon(1-29)) antagonists. The presence of both receptors was demonstrated by Western blot, immunofluorescence and cytofluorimetric analysis of ASCs. In conclusion, we demonstrated a direct inhibitory action of glucagon on the proliferation and differentiation of human adipose precursors, which seems to involve both GLP-1R and GCGR. These findings suggest that the adipose stem compartment is a novel target of glucagon, possibly contributing to the weight loss obtained in vivo with dual GLP-1R/glucagon agonists.
\end{abstract}

\section{Introduction}

Obesity is one of the most critical risk factor for the development of type 2 diabetes (T2D); in fact, about $90 \%$ of persons with diabetes is obese or overweight (Pappachan et al. 2017). This led to the use of the term 'diabesity', indicating such a strong association between the two pathologies (Astrup \& Finer 2000).
Among glucose-lowering drugs used for the treatment of T2D, glucagon-like peptide-1 (GLP-1) receptor agonists (GLP-1RAs) reduce body weight and adiposity both in T2D and in non-diabetic obese subjects (Monami et al. 2012, Pi-Sunyer et al. 2015, Cantini et al. 2016). One of the GLP-1RAs, liraglutide, has recently been approved for 
the treatment of obesity. In addition, a 4-week-treatment of healthy and obese subjects with oxyntomodulin, an endogenous enteroendocrine peptide that acts as a dual agonist for GLP-1R and glucagon receptor (GCGR), reduced food intake and enhanced energy expenditure, thus resulting in a significant body weight loss (Wynne et al. 2005, Bagger et al. 2015). Consistently, pharmacological dual agonists acting on GLP-1R/GCGR have shown a greater efficacy in inducing weight loss in diet-induced obese mice in comparison with specific GLP-1R agonists (Pocai et al. 2009). Encouraging results have also been obtained in humans with the use of GLP-1/ glucagon dual agonists combining the lipolytic activity of glucagon with the glucose-dependent insulinotropic and weight loss action of GLP-1, without inducing glucagonassociated hyperglycemia (Sánchez-Garrido et al. 2017). Compared to placebo, the dual agonist AR425899 significantly reduced fasting plasma glucose and glycated haemoglobin, together with body weight, in patients with T2D (Tillner et al. 2019). However, presently available data are still insufficient to establish whether dual GLP-1R/ GCGR agonists are more effective than the single GLP-1 agonists in the treatment of obesity and diabetes.

In an in vitro cell model of human adipose precursors (ASCs) derived from abdominal fat depots and characterized by our group (Baglioni et al. 2009, 2012), liraglutide and GLP-1 have recently been demonstrated to interfere with adipocyte proliferation and differentiation (Cantini et al. 2015, 2017), as further confirmed by El Bekay and colleagues (El Bekay et al. 2016). These effects seem to be mediated by the classical GLP-1 receptor, as they are reverted by the GLP-1R antagonist exendin 9-39 (Cantini et al. 2015, 2017). However, similar effects have also been obtained with the DPP4-cleaved form GLP-1 (9-36), which displays a reduced binding for the classical GLP-1R; the effects of GLP-1 (9-36) are not reversible by exendin 9-39 (Cantini et al. 2017). All together, these findings suggest the involvement of alternative mechanisms in mediating GLP-1 effects in ASCs (Cantini et al. 2016).

Glucagon, a 29 amino acid peptide produced by the enzymatic cleavage exerted by the prohormone-convertase 2 on the same proglucagon precursor generating GLP-1 and oxyntomodulin, is secreted from the pancreatic alpha cells under hypoglycaemic conditions to restore blood glucose levels (Habegger et al. 2010). Its secretion is finely regulated by intestinal peptides, such as GLP-1, oxyntomodulin and glucose-dependent insulinotropic peptide (GIP), but also by glucagon itself in a paracrine way (Wewer et al. 2016, Müller et al. 2017). Glucagon reduces food intake in rodents and humans; moreover, a sustained activation of GCGR has been demonstrated to enhance lipolysis (Day et al. 2009), although underlying mechanisms are still unclear.

The present study assessed the direct effects of glucagon on the stem cell compartment of human adipose tissue, characterizing the receptors involved.

\section{Materials and methods}

\section{Materials}

Media and sera for cell cultures were purchased from Sigma-Aldrich and tissue plastic ware was obtained from Corning (Milan, Italy). AdipoRed was from Lonza (Milan, Italy). Glucagon, exendin (9-39), [des-His1-Glu9-glucagon (1-29)] and GLP-1(7-36) were purchased from SigmaAldrich, as well as the other reagents for cell cultures, fluorescence microscopy, except where differently indicated.

\section{Human adipose-derived stem (ASC) cell populations}

Abdominal adipose stem cell populations (ASCs) previously isolated and characterized (Baglioni et al. 2009, 2012) from nine morbidly obese subjects (two males and seven females: mean age \pm s.D.: $54 \pm 13$ years; BMI \pm s.D.: $\left.39.2 \pm 7.7 \mathrm{~kg} / \mathrm{m}^{2}\right)$, who underwent bariatric surgery at Careggi University Hospital (following written informed consent, Local Ethical Committee's approval Ref. Protocol 58-11), have been used in this study. ASCs were cultured in DMEM containing 20\% fetal bovine serum (FBS), $100 \mathrm{mg} / \mathrm{mL}$ streptomycin, $100 \mathrm{U} / \mathrm{mL}$ penicillin, $2 \mathrm{mM}$ L-glutamine, and $1 \mu \mathrm{g} / \mathrm{mL}$ amphotericin-B, at $37^{\circ} \mathrm{C}$ in humidified atmosphere with $5 \% \mathrm{CO}_{2}$, and used between passages 2 and 6 . Each experiment was performed at least three times using ASCs derived from at least three independent subjects. In all experiments, glucagon, GLP-1 and receptors' antagonists have been added from the beginning and freshly re-added every 3 days.

\section{ASC viability and proliferation assay}

\section{Cell count}

Seeded cells $\left(7 \times 10^{3}\right)$ were treated with glucagon (1-10$100 \mathrm{nM})$ for the indicated times, and then trypsinized and counted in the haemocytometer. Mean cell number was obtained by counting at least four replicates for each point in each experiment. Dead cells were excluded by trypan blue test. 


\section{DNA synthesis assay - $\left[{ }^{3} \mathrm{H}\right]$ thymidine uptake}

DNA synthesis was evaluated according to the amount of $\left[{ }^{3} \mathrm{H}\right]$ thymidine (Perkin Elmer) incorporated into trichloroacetic acid (TCA)-precipitated materials. Cells grown until $70 \%$ confluence and $24 \mathrm{~h}$-starved, were treated with glucagon (10 and $100 \mathrm{nM}$ ) for 24 and $48 \mathrm{~h}$, pulsing them with $0.5 \mu \mathrm{Ci} / \mathrm{mL}\left[{ }^{3} \mathrm{H}\right] \mathrm{TdR}(6.7 \mathrm{Ci} / \mathrm{mmol})$ for $4 \mathrm{~h}$ before stopping proliferation in 10\% ice-cold TCA. After washing in 5\% TCA, cells were solubilized in $0.25 \mathrm{~N} \mathrm{NaOH}$, at $37^{\circ} \mathrm{C}$ and radioactivity was measured on a scintillation beta-counter (Tri-Carb 2800TR Liquid Scintillation Analyzer, Perkin Elmer). Experiments were performed in quadruplicate in three independent cell populations.

\section{Cytofluorimetric cell count}

Seven thousand ASCs seeded in 12-well plates and grown at confluence were treated with glucagon (1-10$100 \mathrm{nM})$. At different time points $(24,48,72 \mathrm{~h})$ cells were trypsinized and analysed with a Muse ${ }^{\mathrm{TM}}$ automated cell analyser (Merck Millipore) using Muse ${ }^{\mathrm{TM}}$ Count \& Viability (cat\#MCH100102, Merck Millipore) assay, according to the manufacturer's instructions.

\section{Cytofluorimetric apoptotic evaluation}

After 24, 48 and 72-h treatment with glucagon (10 and $100 \mathrm{nM})$, ASCs were trypsinized and analysed with a Muse $^{\mathrm{TM}}$ automated cell analyser (Merck Millipore) for the early apoptosis detection using Muse ${ }^{\mathrm{TM}}$ Annexin V/Dead Cell (cat\#MCH100105, Merck Millipore) assay, according to the manufacturer's instructions.

\section{Cytofluorimetric cell cycle analysis}

ASCs treated for 24 and $48 \mathrm{~h}$ with glucagon (10 and $100 \mathrm{nM}$ ) were trypsinized and analysed with a Muse ${ }^{\mathrm{TM}}$ automated cell analyser (Merck Millipore) for the evaluation of cell cycle using Muse ${ }^{\mathrm{TM}}$ Cell Cycle kit (cat\#MCH100106, Merck Millipore) assay, according to the manufacturer's instructions.

\section{In vitro-adipose differentiation}

ASCs ( $10^{5}$ cells) were cultured in a 6-well plate in $10 \%$ FBS-DMEM, $0.5 \mathrm{mM} 3$-isobutyl-1-methylxanthine, $1 \mu \mathrm{M}$ dexamethasone, $200 \mu \mathrm{M}$ indomethacin and $10 \mu \mathrm{M}$ insulin for 2 weeks, and then shifted to 10\% FBS-DMEM containing $1.7 \mu \mathrm{M}$ insulin for another week (Baglioni et al. 2009, 2012). Glucagon and receptors' antagonists have been added from the beginning of adipose differentiation. Stimuli have been freshly re-added every 3 days.

\section{Intracellular triglyceride staining techniques}

\section{Oil Red 0 - AdipoRed}

Adipose differentiation was assessed by Oil Red $\mathrm{O}$ or AdipoRed staining of intracellular neutral lipids in in-vitro-differentiated adipocytes, observed under optical or fluorescence microscopy, respectively.

\section{AdipoRed staining of intracellular lipid droplets}

ASCs grown on plastics or on glass coverslips were subjected to adipose differentiation in the absence or presence of glucagon (1-10-100 nM), and then were treated with the AdipoRed staining as described earlier (Cantini et al. 2015). Quantitative spectrofluorimetric detection of AdipoRed fluorescence emission was measured with an ELISA plate reader (Wallac 1420, Perkin Elmer) at $485 / 572 \mathrm{~nm}$ excitation/emission. Specific absorbance of the intracellular lipid droplets in differentiated adipocytes was calculated as fold increase on unspecific absorbance of undifferentiated ASCs used as control, after the blank subtraction. This value correlates with the entity of differentiation (number and size of mature adipocytes formed). Each point has been obtained in ten replicates from five independent experiments.

The AdipoRed assay is referred to the total intracellular accumulation of triglycerides in all differentiated adipocytes and is not considered a measurement of the single cell intracellular lipid content.

After fixation in $4 \%$ paraformaldehyde, cells were counterstained with DAPI ((1:2000), Sigma-Aldrich) and the coverslips mounted using the ProLong ${ }^{\circledR}$ Gold Antifade Mountant (ThermoFisher Scientific). Fluorescence images were acquired with a Leica DM4000 epifluorescence microscope (Leica Microsystems GmbH).

\section{mRNA isolation and quantitative real-time RT-PCR}

mRNA isolation from cells was performed as previously detailed (Baglioni et al. 2009, 2012) and quantitative realtime RT-PCR (qRT-PCR) was carried out using primers and probes for FABP4, HSL, PPAR $\gamma$ and GAPDH genes (Taqman Gene Expression Assay, Life Technologies, respective codes: HS00609791_m1, Hs00193510_m1, Hs00234592_m1, FAM-MGB 4325934-1301038). The amount of target, normalized to the endogenous reference gene (GAPDH) and relative to a calibrator (Stratagene, La Jolla, CA, USA) was calculated by $2^{-\Delta \Delta C t}$. 


\section{SDS-PAGE and Western Blot analysis}

ASC proteins were extracted in RIPA buffer ( $20 \mathrm{mM}$ Tris, $\mathrm{pH}$ 7.4, $150 \mathrm{mM} \mathrm{NaCl}, 0.5 \%$ Triton-100, $1 \mathrm{mM} \mathrm{Na} \mathrm{VO}_{4}, 1 \mathrm{mM}$ PMSF). Thirty micrograms of proteins were separated by reducing SDS-PAGE stain-free precast gels ${ }^{\circledR}$ (Bio-Rad) and transferred to PVDF membranes (Trans-Blot ${ }^{\circledR}$ TurboTM, Bio-Rad).

Membranes were probes with the following primary antibodies: polyclonal anti-GLP-1R (1:200, sc-34637; Santa Cruz Biotechnology), monoclonal anti-GLP-1R (1:1000, sc-390773; Santa Cruz Biotechnology), polyclonal anti-GLP-1R (1:500, ab186051; Abcam), anti-glucagon receptor (1:200, sc34641; Santa Cruz Biotechnology). Each membrane was incubated overnight at $4^{\circ} \mathrm{C}$ with primary antibodies followed by peroxidase-conjugated secondary IgGs (1:2000). Image acquisition and analysis were performed with Image Lab software on a ChemiDoc TM Touch instrument (Bio-Rad), using fluorescence emission of protein bands separated on stain-free gels for the total lane normalization. All Western blot experiments were repeated at least three times.

\section{GLP-1R/GCGR detection in ASCs by flow cytometry analysis}

Analysis of GLP-1R/GCGR expression in ASC populations was performed by using the FITC-conjugated monoclonal/ polyclonal antibodies and their respective negative controls (secondary antibodies, Sigma-Aldrich). Cells were acquired from each sample on FACScan flow cytometer (Becton-Dickinson) and green fluorescence was detected by the FL1 (515-555 nm wavelength band) detectors. Flow cytometric analysis was stopped when 1000 events were acquired.

\section{Fluorescence Immunocytochemistry}

Ten thousand cells grown on glass coverslips in a six-well plate were fixed with $4 \%$ paraformaldehyde, for $10 \mathrm{~min}$, at room temperature. PBS-washed coverslips were blocked in $5 \%$ goat serum, and then incubated for $2 \mathrm{~h}$ at room temperature with the monoclonal anti-GLP1R (1:50, sc-390773), polyclonal anti-GLP-1R (1:50, ab186051), or anti-glucagon receptor (1:20, sc34641) diluted in 5\% goat serum. Coverslips were incubated with an FITCconjugated anti-goat or anti-mouse IgG antibody (1:100, Sigma-Aldrich) for $1 \mathrm{~h}$, and then were mounted using the ProLong® Gold Antifade Mountant with DAPI (\#P36962, ThermoFisher Scientific).
Images were acquired with a Leica DM4000 epifluorescence microscope (Leica Microsystems $\mathrm{GmbH}$ ). Negative controls (not shown) were performed avoiding primary antibodies.

\section{Statistical analysis}

Statistical analysis was performed using SPSS 25.0 software (SPSS Inc.). The Kolmogorov-Smirnov test was used to verify the normal distribution of data, which was expressed as mean \pm s.e. One-way ANOVA followed by post hoc Dunnett's test was applied for multiple comparisons, while Student's $t$ test was applied for analysis of two classes of data. A $P<0.05$ value was considered statistically significant.

\section{Results}

\section{Glucagon exerts an anti-proliferative effect on human adipose precursor cells}

The in vitro administration of increasing concentrations of glucagon (1-10-100nM) resulted in a reduction in the number of human ASCs. ASC exponential growth was significantly inhibited by glucagon, in a dose- and timedependent manner, with the maximal effect for the highest dose (37\%), as evaluated by direct cell count, observed in the third day (Fig. 1A). The decrease in cell number following glucagon treatment was confirmed by FACScan analysis, which highlighted a significant increase in the number of dead cells, starting from day 1 with glucagon $100 \mathrm{nM}$; this reduction was statistically significant for the lower (10nM) dose at days 2 and 3 (Fig. 1B and C). A significant reduction in the proliferation rate in response to glucagon (10-100 nM) was demonstrated through thymidine incorporation assay (Fig. 1D). This finding was confirmed by a significant reduction in the number of cells in the $S$-phase of the cell cycle (analysed by FACScan) following 24-h glucagon exposure (14 and 19\% for 10-100nM glucagon) and 48-h glucagon (25\% for both doses) (Fig. 1E and F). No significant effect of glucagon on cell apoptosis, as evaluated by cytofluorimetric evaluation of annexin $\mathrm{V}$ exposure, was observed at any time points (Fig. 2A and B).

\section{Glucagon inhibits in vitro-induced adipogenesis}

To assess glucagon effects on the adipogenic potential of adipose precursors, cultured ASCs were in vitrodifferentiated in the presence of increasing concentrations of glucagon (1-10-100nM). AdipoRed and Oil Red O staining of intracellular triglyceride depots, observed under 


\begin{tabular}{|l|l|l|l|l|}
$\begin{array}{l}\text { Journal of Molecular } \\
\text { Endocrinology }\end{array}$ & G Cantini et al. & $\begin{array}{l}\text { Glucagon effect in adipose } \\
\text { stem cells }\end{array}$ & $\mathbf{6 3 : 4}$ & $\mathbf{2 5 3}$ \\
\hline
\end{tabular}

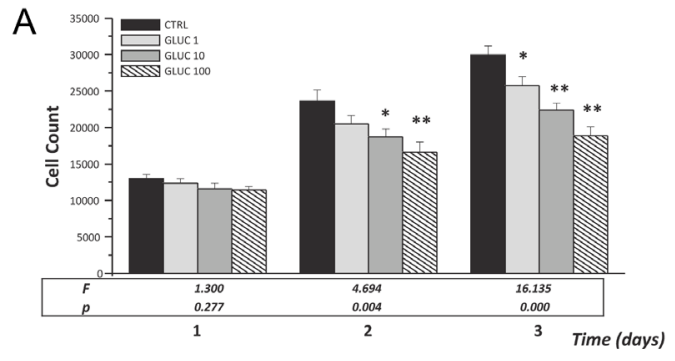

B
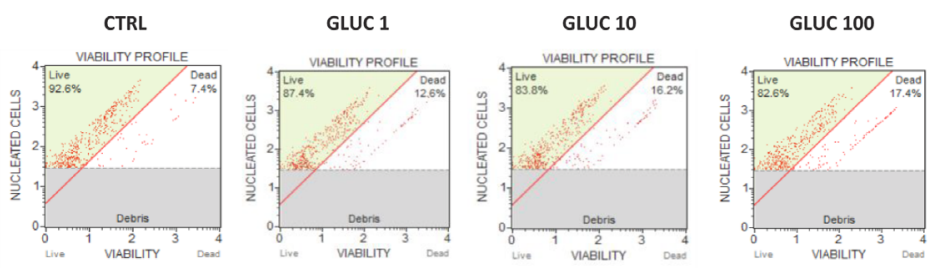

C
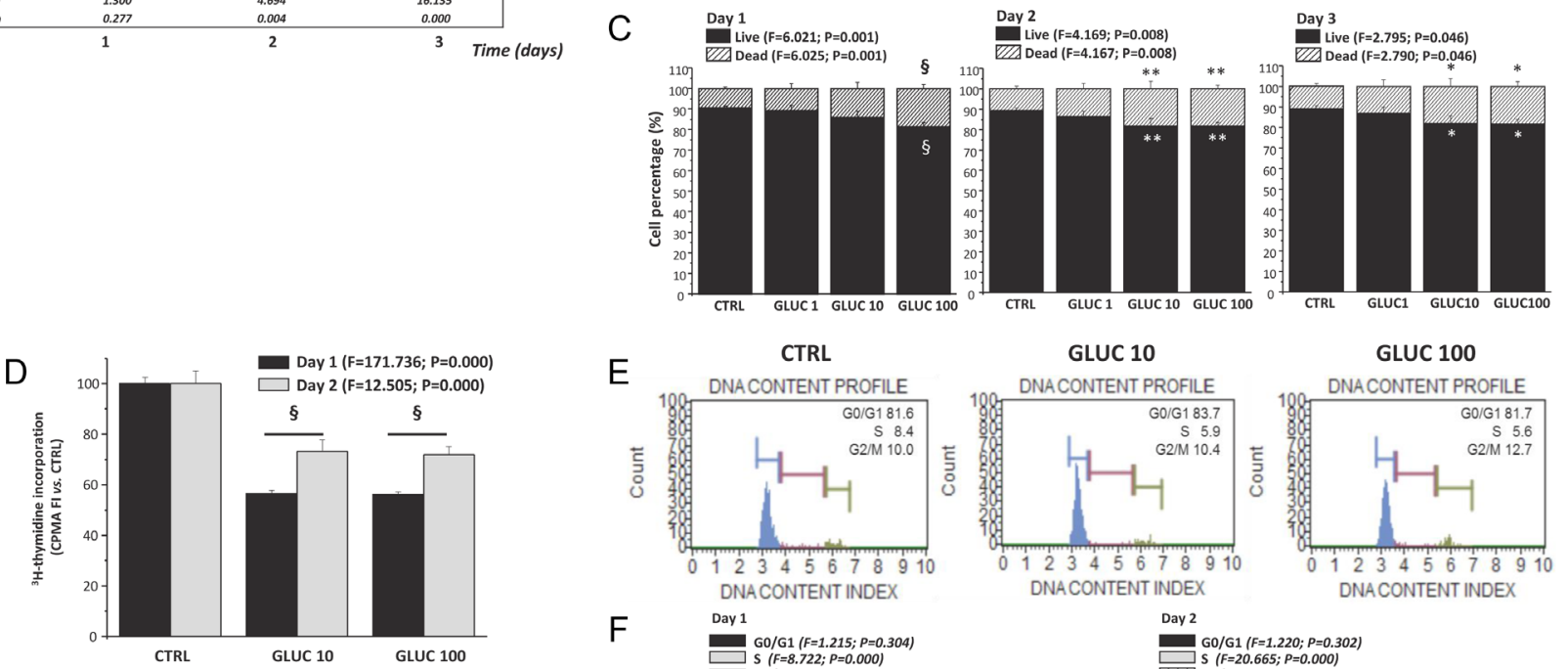

$\mathrm{F}$

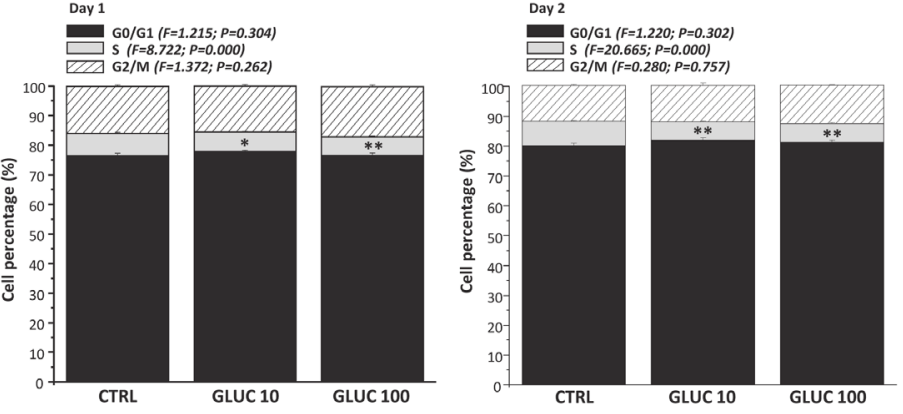

\section{Figure 1}

The anti-proliferative action of glucagon in adipose precursors. ASCs were untreated (CTRL) or treated for 1, 2 or 3 days with increasing concentration (1-10-100 nM) of glucagon. (A) Haemocytometer cell-counting results are expressed as mean \pm S.E. (B) Representative scatter plots of cytofluorimetric evaluation of cell viability are shown; (C) bar-graphs represent mean \pm S.E. of cell percentages. (D) Data represent mean \pm S.E. cpm after a 4-h pulse of $\left[{ }^{3} \mathrm{H}\right]$-thymidine in ASCs untreated/treated for 24-48 h with 10-100 nM glucagon. (E) Representative scatter plots of DNA content index are presented; (F) data obtained from cell cycle analysis are indicated as mean \pm S.E. of cell percentage in ASCs untreated/treated for 24-48 h with 10-100 nM glucagon. Statistical analysis was performed with ANOVA ( $F$ and $P$ value are indicated) followed by Dunnett's post hoc test: $* P<0.05, * \star P<0.01, \S P<0.000$ vs respective $C t r l, n=5$ independent experiments.

fluorescence (Fig. 3A, B, C, D, E, F, G and H) or optical (Fig. 3I, J, K and L) microscope, revealed a lower number of differentiated adipocytes with smaller lipid droplets in glucagon-treated wells. The quantitative evaluation of AdipoRed assay confirmed a statistically significant reduction in fluorescence intensity in ASCs differentiated with increasing doses of glucagon (fold decrease vs ADIPO: 1.1, 1.5, 1.4 for glucagon 1-10-100 nM, respectively) Fig. 3M.

In order to confirm the inhibitory effect of glucagon on adipogenesis, gene expression of the specific adipose differentiation markers, the early master gene PPAR $\gamma$ and the fatty acid-binding protein-4 (FABP4) and hormonesensitive lipase $(H S L)$, as marker of mature adipocytes, were quantified by qRT-PCR TaqMan analysis. A significant decrease in the expression level of analysed genes in in vitro-differentiated adipocytes was detected in the presence of glucagon (\% inhibition vs ADIPO: 33, 35, 29\%, for PPAR , 28, 66, 37\% for FABP4 and 46, 54, 37\% for $H S L$, for glucagon 1-10-100 nM, respectively) (Fig. 3N).

\section{ASCs express GLP-1R and GCGR}

In our previous studies, we observed that the antiproliferative and anti-lipogenic effect exerted by liraglutide on ASCs seems to be at least partially mediated by the classical GLP-1R (Cantini et al. 2015). However, the 
A
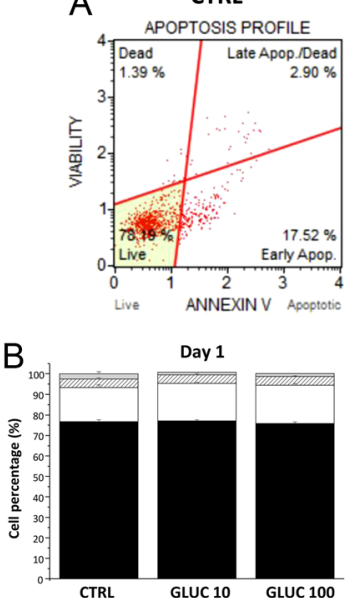

Day 1

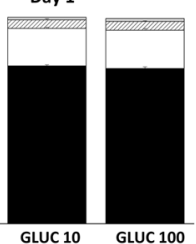

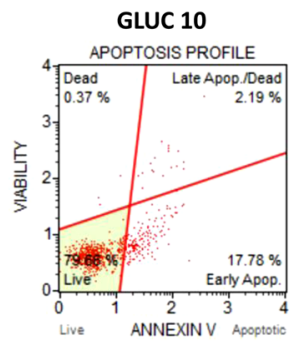

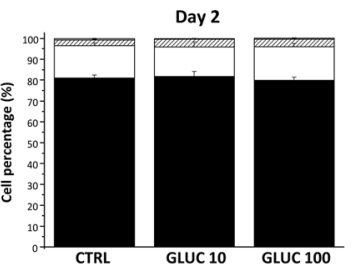

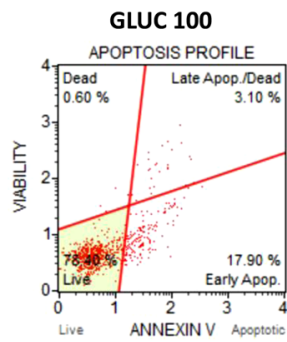

Day 3

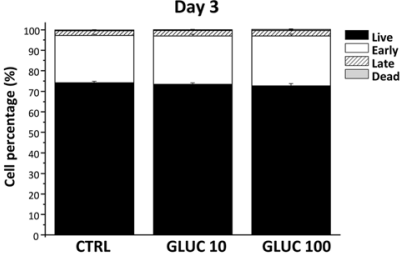

Figure 2

Glucagon stimulation does not affect apoptosis in adipose precursors. ASCs were untreated (CTRL) or treated for 1, 2 or 3 days with increasing concentration (10-100 nM) of glucagon. (A) Representative scatter plots of cytofluorimetric evaluation of annexin $V$ are shown. (B) Bar graphs represent mean \pm S.E. of cell percentages.

Statistical analysis was performed with ANOVA resulting in no statistically significant differences, $n=3$ independent experiments. A full colour version of this figure is available at https://doi. org/10.1530/JME-19-0095. expression of GLP-1R in the adipose tissue and in adipose precursors is still debated (Vendrell et al. 2011, Muscogiuri et al. 2014, Cantini et al. 2016, El Bekay et al. 2016). Therefore, we evaluated the expression of GLP-1R and GCGR in ASCs using different complementary techniques and different monoclonal and polyclonal antibodies. First, we confirmed GCGR and GLP-1R protein expression on the cell surface by immunofluorescence cytochemistry (Fig. 4A). The receptor positivity detected by fluorescence microscopy was confirmed by FACScan evaluation of ASCs using the same antibodies against GCGR and GLP-1R
(Fig. 4B). Finally, the presence of both receptors was detected both in ASCs and in the corresponding adipose tissue (AT) samples by Western blot analysis at the expected molecular weight (Fig. 4C).

\section{An interplay between GCGR and GLP-1R is involved in mediating glucagon effects}

We had previously reported that the effects of GLP1RAs such as liraglutide and native GLP-1(7-37) on human adipose precursors are significantly attenuated
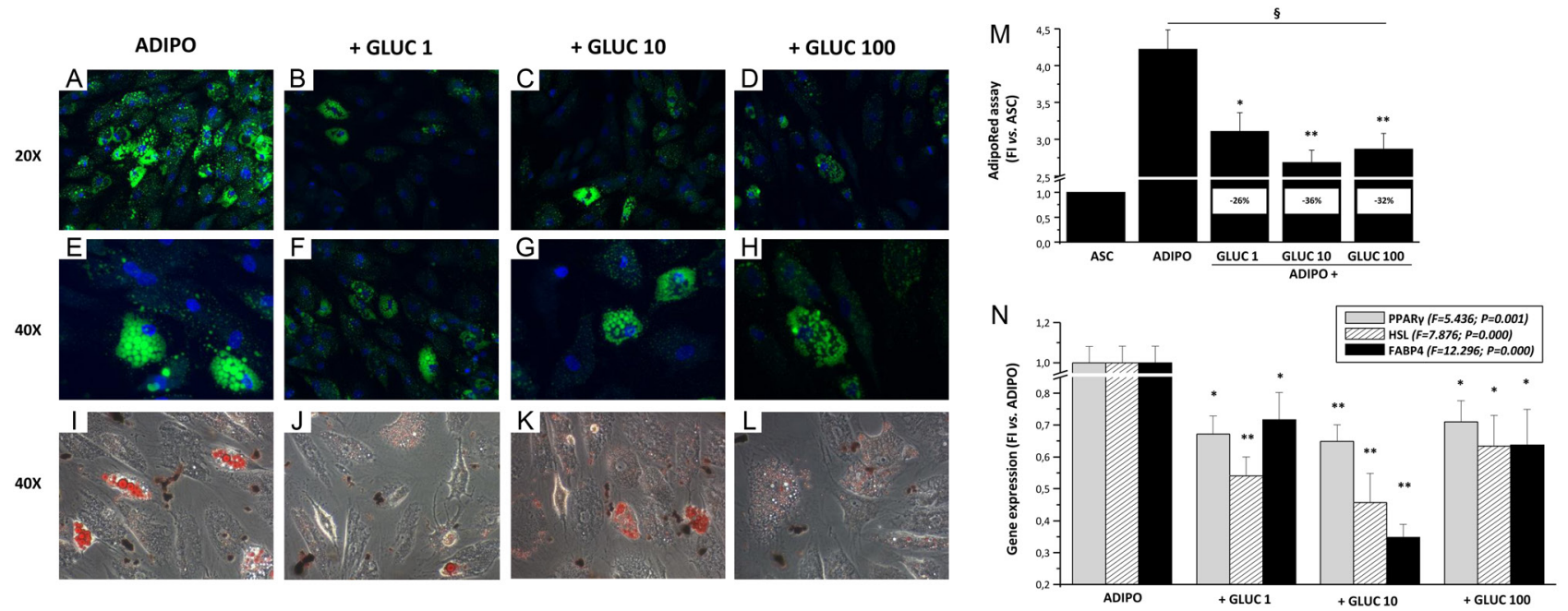

\section{Figure 3}

Glucagon interferes with the in vitro-induced adipose differentiation. ASCs were untreated (ASC) or in vitro induced to differentiate towards the adipose phenotype with the specific inductive media (ADIPO) alone or in the presence of increasing concentration (1-10-100 nM) of glucagon. Representative images of in vitro-derived adipocytes stained with AdipoRed fluorescence reagent (A-H) or Oil Red O staining (I-L), captured by epifluorescence or optical microscopy, respectively. Any Oil Red O or AdipoRed staining was present in undifferentiated ASC controls (not shown). Magnification (20× A-D, 40× E-L). (M) Quantitative evaluation of intracellular triglyceride depots expressed as mean \pm S.E. absorbance fold increase (FI) vs ASC after spectrofluorometric measurement of AdipoRed staining. Percentages of glucagon inhibitory effect in lipid content are indicated. Statistical analysis was performed with ANOVA $(F=5.915 ; P=0.001)$ followed by Dunnett's post hoc test: $\S P<0.000$ vs undifferentiated ASCs, $* P<0.005$, $* \star P<0.000$ vs ADIPO, $n=5$ independent experiments. (N) TaqMan qRT-PCR evaluation of adipose marker gene expression in in-vitro-differentiated adipocytes alone or treated with increasing concentration (1-10-100nM) of glucagon: bar-graph represents mean \pm s.E. gene expression fold increase (FI) vs ADIPO. Statistical analysis was performed with ANOVA followed by Dunnett's post hoc test: $* P<0.05, * \star P<0.001$ vs ADIPO, $n=5$ independent experiments. 

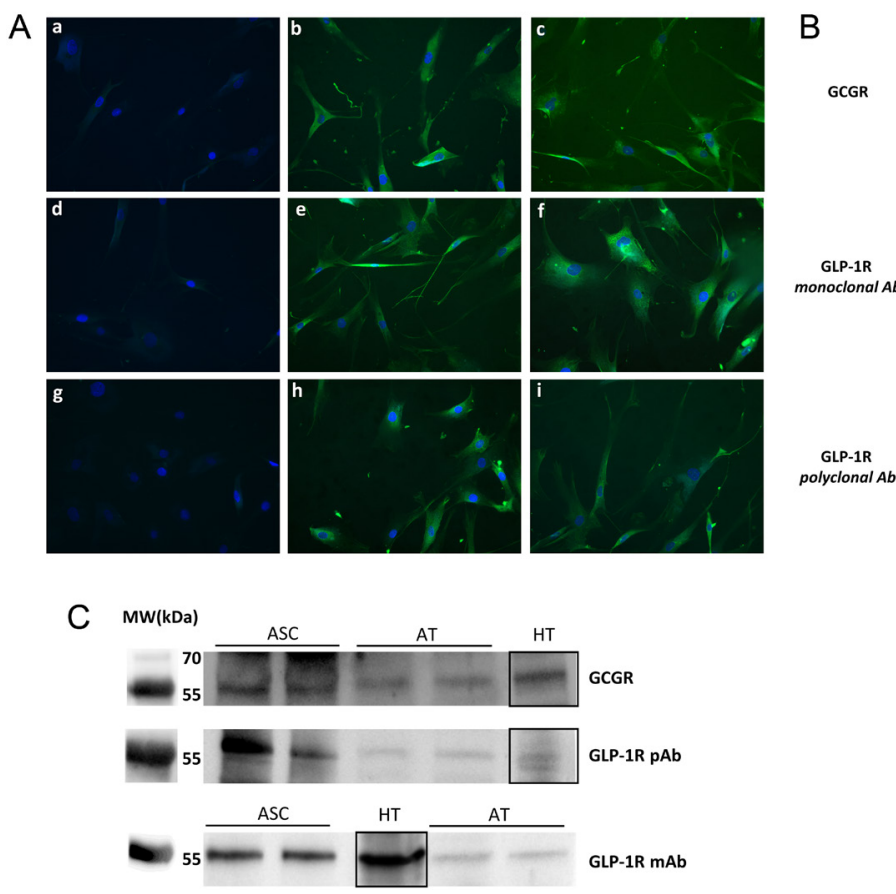

or completely reverted in the presence of the specific GLP-1R antagonist exendin 9-39 (Cantini et al. 2015, 2017). An inhibitory effect similar to that observed with liraglutide had been observed with the cleaved GLP-1 form, GLP-1(9-36), which displays a reduced binding ability to the classical receptor. In order to better clarify the role of GLP-1R and GCGR in mediating the similar effects observed with GLP-1RAs and glucagon in ASCs, we used the specific glucagon receptor antagonist, des-His1[Glu9]-glucagon(1-29) (GI) (Unson et al. 1987, Post et al. 1993, Chepurny et al. 2019), along with the antagonist of GLP-1R exendin 9-39 (EX). When cell count experiments were repeated in the presence of glucagon alone or in combination with GI $(10 \mathrm{nM})$ or EX $(10 \mathrm{nM})$, the inhibitory effect exerted by glucagon on ASC proliferation was significantly reverted not only by GI $(86,103,97 \%$ for $24,48,72$-h treatment, respectively), but also by EX (88, 96, 91\% for 24, 48, 72-h treatment, respectively), Fig. 5A. Similarly, the inhibitory effect exerted by GLP-1 on ASC proliferation was significantly dampened and reversed in the presence of GI $(92,104,92 \%$ for 24, 48, 72-h treatment, respectively), Fig. 5B. The engagement of both receptors in mediating the effect of glucagon on cell proliferation was further supported by the absence of any synergistic effect between glucagon and GLP-1 when added to the cells at the equimolar dose of $10 \mathrm{nM}$ (Fig. 5C).

When the two antagonists of GLP-1R and GCGR were added with glucagon during in vitro-induced adipose differentiation (GI and exendin 9-39, 10 nM), a significant
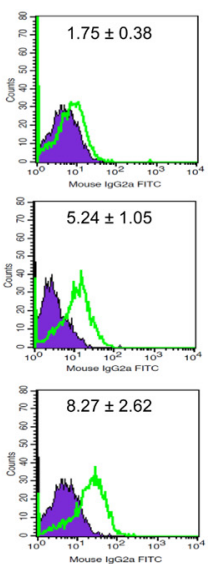

\section{Figure 4}

GCGR and GLP-1R expression in human adipose precursor cells. (A) ASCs were subjected to an immunocytochemistry using the different antibodies for GCGR and GLP-1R, as specified in the methods section. Representative images of cells incubated with GCGR primary antibody (panels $b$ and $c$ ), monoclonal (panels e-f) and polyclonal (panels h-i) primary GLP-1R antibodies and visualized on epifluorescence microscopy are shown. Negative controls (panels a-d-g) avoiding the primary antibody are shown. Nuclear counterstaining with DAPI is presented (blue signal). Magnification is 20×. (B) Cytofluorimetric evaluation of GCGR and GLP-1R on cell surface. Representative scatter plots are shown: data is expressed as the mean \pm S.D. of positive cells in $n=3$ independent experiments. (C) Western blot analysis of GCGR and GLP-1R expression in different ASC populations compared to the positive controls (AT, adipose tissue and HT, heart samples). Protein ladder bands are shown in the left. reversion of glucagon inhibition of adipogenesis was observed with both antagonists, as shown by AdipoRed intracellular lipid quantitative staining (reversion percentage of 79 and 93\% for GI and EX, respectively), Fig. 6A. These findings were further confirmed by the reversion of glucagon inhibition of FABP4 and HSL gene expression observed in the presence of each antagonist (GI: 88 and 77\% for FABP4 and HSL, respectively; exendin 9-39: 104 and 95\% for FABP4 and HSL, respectively) (Fig. $6 \mathrm{~B})$. The simultaneous addition of the two receptor's antagonists together with glucagon showed a statistically significant reversion of the adipogenesis inhibition higher than those observed with single antagonist stimulation (149 and $133 \%$ vs GI or EX alone), as assessed by quantitative evaluation of FABP4 gene expression (Fig. 6C). The percentage of FABP4 expression versus ADIPO was 95, 110, 126\% for GI, EX and GI+EX, respectively (Fig. 6C). Taken together these findings suggested that the combination of inhibitors totally recovered the inhibitory effect of glucagon even to a higher extent than the single antagonist.

\section{Discussion}

Although prior pharmacological approaches to obesity had failed to achieve and maintain in the long-term a relevant weight loss, some GLP-1R agonists used for the treatment of T2D have been recently developed as anti-obesity drugs. In order to improve the effects on weight of GLP-1 

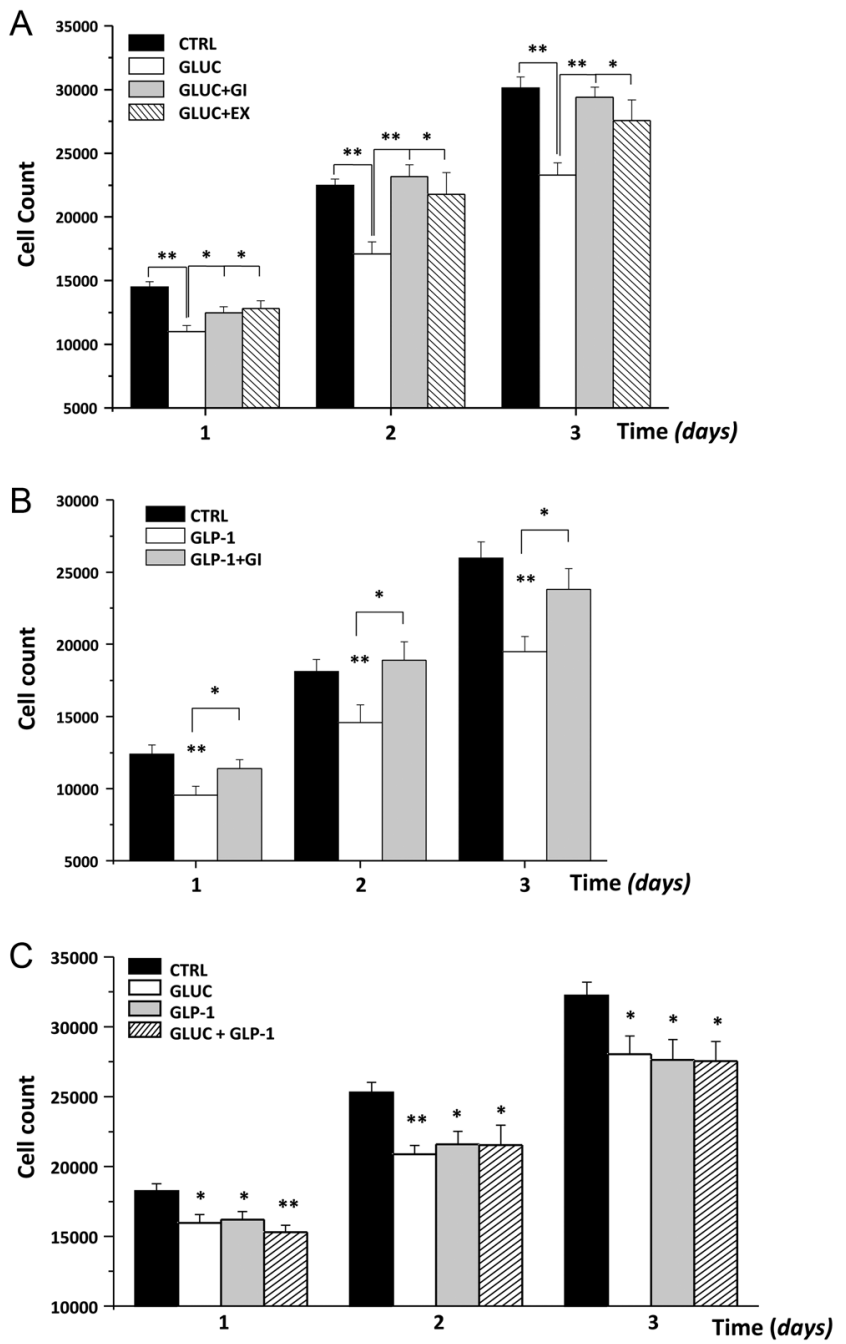

\section{Figure 5}

GCGR and GLP-1R involvement underlying the anti-proliferative effect of glucagon in human adipose precursor cells. (A) ASCs were untreated (CTRL) or treated for 1,2 or 3 days with $10 \mathrm{nM}$ glucagon alone or combined with an equimolar concentration $(10 \mathrm{nM})$ of the GCGR antagonist, des-His1-Glu9-glucagon(1-29) (GI) or the GLP-1R antagonist exendin 9-39, (EX): results are expressed as mean \pm s.E. of direct cell count. (B) ASCs were untreated (CTRL) or treated for 1, 2 or 3 days with $10 \mathrm{nM}$ GLP-1 alone or combined with an equimolar concentration (10 nM) of the GCGR antagonist, des-His1-Glu9-glucagon(1-29) (GI): quantitative results are expressed as mean \pm S.E. of direct cell count. (C) ASCs were untreated (CTRL) or treated for 1,2 or 3 days with $10 \mathrm{nM}$ glucagon (GLUC) alone or combined with an equimolar concentration (10 nM) of GLP-1: results are expressed as mean \pm S.E. of direct cell count. Statistical analysis was performed with Student's $t$ test: ${ }^{*} P<0.05, * * P<0.01, n=3$ independent experiments.

(Tan et al. 2013, Tillner et al. 2019), the use of glucagon in association with GLP-1 (Day et al. 2009) or of dual agonists for both GLP-1 and glucagon receptors (Pocai et al. 2009, Tillner et al. 2019) seems to be a promising approach. Specific research in this field has raised a novel interest in the systemic catabolic properties of glucagon, besides its well-known role in regulating glucose homeostasis as counter-actor of insulin. In in vivo mouse models of obesity, treatment with dual agonist determined enhanced weight loss and a greater improvement in several metabolic parameters in comparison with selective GLP-1 agonists (Pocai et al. 2009). Indeed, glucagon-induced suppression of appetite and increase of energy expenditure were described a long time ago, together with the stimulation of catabolic processes alternative to glucose consumption, such as amino acid and lipid catabolism, to maintain the fuel for the organism and carbonic skeletons to support gluconeogenesis (Ahrén 2015).

We show here novel direct effects of glucagon on the stem compartment of human AT, in addition to the stimulation of lipolysis already known for mature adipocytes (Bertin et al. 2001, Arafat et al. 2013). Glucagon administration to human primary ASC significantly inhibits cell proliferation, reducing cell precursor number and in vitro-induced adipogenesis; this is demonstrated not only by a decrease in intracellular lipid droplet accumulation but also in a lower expression of mature adipocyte markers, such as $H S L$ and FABP4 and the early master gene, such as $P P A R \gamma$. These effects may contribute to the weight loss observed in treated subjects through a direct action on the AT. Notably, our results suggest that glucagon could act not only exerting a direct action on the mature adipocytes, but also affecting the expansion of the adipose compartment, by interfering more precociously on the number of precursors undergoing adipogenesis (limiting their proliferation and reducing the number of mature adipocytes formed), and thus resulting in a longer lasting effect. The inhibition of recruitment and differentiation of precursors into adipocytes might lead to hypertrophy and dysfunction in adipose cells or to ectopic accumulation of lipids in other depots, leading to the development of insulin resistance. This specific issue, which would require in vivo animal studies, was not within the aim of the present study. However, available data on dual agonists for GLP-1 and glucagon receptors have so far excluded any detrimental effect on insulin resistance; conversely, they showed a beneficial effect on blood glucose beside weight loss (Pocai et al. 2009, Tan et al. 2013, Sánchez-Garrido et al. 2017, Tillner et al. 2019).

We had recently demonstrated that liraglutide (Cantini et al. 2015), and to a lesser extent native GLP-1 (Cantini et al. 2017), exert similar effects in the same adipose precursor cell model at the equimolar concentration of $10 \mathrm{nM}$. Both liraglutide (Cantini et al. 2015) and glucagon (as we show in the present paper) reduce ASC number by inhibiting cell proliferation and 

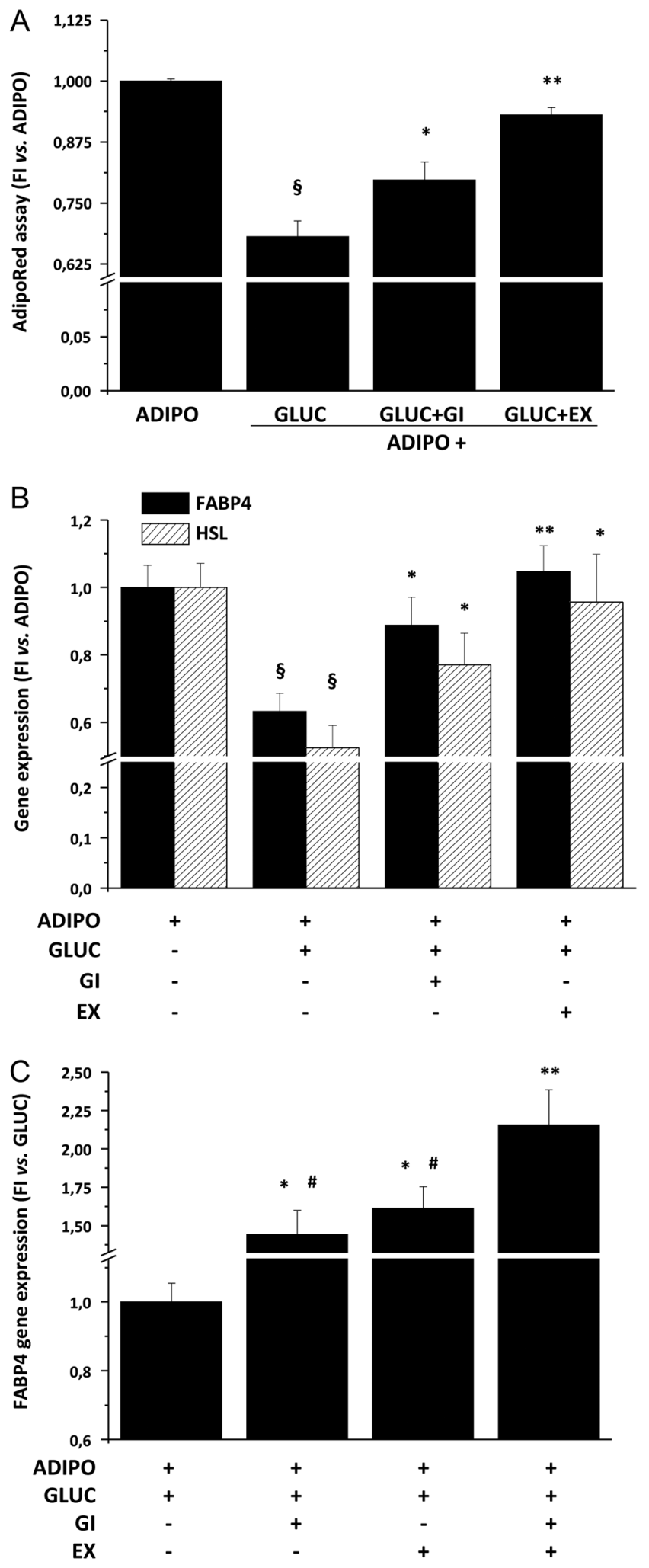

Figure 6

GCGR and GLP-1R involvement underlying the anti-adipogenic effect of glucagon in human adipose precursor cells. ASCs were untreated (ASC) or in vitro induced to differentiate towards the adipose phenotype with the specific inductive media (ADIPO), with $10 \mathrm{nM}$ glucagon (GLUC) or

(c) 2019 Society for Endocrinology Published by Bioscientifica Ltd. Printed in Great Britain inducing cell death. However, liraglutide only was able to stimulate cell apoptosis, whereas glucagon seemed to induce cell death in a non-apoptotic manner. In particular, the significant reduction in the proliferation rate (of about $40 \%)$ measured by thymidine incorporation at the two highest doses of glucagon (10-100 nM) seems to be due to a reduction in the number of cells entering the S-phase.

Despite the opposite contribution of glucagon and GLP-1 in the control of glucose homeostasis, a convergence of action of the two molecules is not surprising, as they share large sequences of homology and derive from alternative post-translational splicing from the same encoding gene. A further suggestive clue supporting the analogy in the activities of the two molecules is represented by their respective receptors, GCGR for glucagon and GLP-1R for GLP-1. Both receptors belong to the G-protein-coupled receptors type $B$ (GPCR) family and are evolutionarily derived from the same ancestor gene through sequential phenomena of duplication and differentiation in paralogue structures coevolving together with their ligands (Irwin \& Wong 2005, Hwang et al. 2014).

GLP-1 mainly acts through the classical GLP-1R (Cantini et al. 2016); however, the expression of the receptor in the AT and in the adipose-derived precursor cells still remains debated (Vendrell et al. 2011, Muscogiuri et al. 2014, Cantini et al. 2015, 2017, El Bekay et al. 2016). GLP-1R expression has also been shown in human bone marrow-derived mesenchymal stem cells (Sanz et al. 2010, Lee et al. 2015). Here, we demonstrate by complementary techniques the expression of both GLP-1R and GCGR at protein level in human adipose precursors and in the corresponding AT.

combined with an equimolar concentration (10 nM) of GCGR (GI) or GLP-1R (EX) antagonists. (A) Quantitative evaluation of intracellular triglyceride depots expressed as mean \pm s.E. absorbance fold increase (FI) vs ADIPO after spectrofluorometric measurement of AdipoRed staining. Statistical analysis was performed with Student's $t$ test: $P<0.000$ vs undifferentiated ASC, $\S P<0.001$ vs ADIPO, $* P<0.05, * * P<0.001$ vs Gluc10, $n=7$ independent experiments. (B) qRT-PCR TaqMan evaluation of adipose marker gene expression in in-vitro-differentiated adipocytes alone (ADIPO), treated with $10 \mathrm{nM}$ glucagon (GLUC) or combined with an equimolar concentration (10 nM) of GCGR (GI) or GLP-1R (EX) antagonists. Bar-graph represents mean \pm S.E. gene expression fold increase (FI) vs ADIPO. Statistical analysis was performed with Student's $t$ test: $P<0.000$ vs undifferentiated ASC, $\S P<0.001$ vs ADIPO, $* P<0.05, * \star P<0.001$ vs GLUC, $n=6$ independent experiments. (C) qRT-PCR TaqMan evaluation of the adipose marker gene expression (FABP4) in in-vitro-differentiated adipocytes treated with $10 \mathrm{nM}$ glucagon (GLUC) stimulated with an equimolar concentration (10 nM) of GCGR (GI) or GLP-1R (EX) antagonists alone or combined. Statistical analysis was performed with Student's $t$ test: $P<0.000$ vs undifferentiated ASC and $P<0.001$ vs ADIPO (not shown), $* P<0.05, * * P<0.001$ vs GLUC, $\# P<0.05$ vs GI + EX combined, $n=4$ independent experiments from two ASC populations. 
To make this scenario even more complex, the ability of glucagon to bind to and activate GLP-1R has been recently demonstrated in pancreatic beta-cells; in these cells, in conditions of high glucose, glucagon can stimulate insulin secretion through a specific binding to beta-cell GLP-1R (Svendsen et al. 2018). As mentioned earlier, such a 'promiscuity' of different GPCRs is further supported by evolutionary studies. It has been reported that some fishes (e.g., zebrafish) do not express a GLP-1R; instead, they have a related receptor, zfGPCR, with dual ligand selectivity for glucagon and GLP-1. The particular affinity of this receptor for the two ligands is due to the absence of the loop 3 in N-terminal extracellular domain of the receptor (NECD), which is responsible for ligand selectivity (Oren et al. 2016). Since we demonstrated the coexistence of both GCGR and GLP-1R in ASCs, we were interested in clarifying which receptor mediated the glucagon effects observed in the present study. To this purpose, we used the best available orthosteric antagonists for GCGR, desHis1-[Glu9]-glucagon (Unson et al. 1987) and exendin 9-39 for GLP-1R (Thorensen et al. 1993), whose selectivity and specificity have been demonstrated in other systems (Unson et al. 1991, Post et al. 1993, Chepurny et al. 2019). The inhibitory effect exerted by glucagon on ASC proliferation was completely reverted by des-His1-[Glu9]glucagon (GI) and, to a lesser extent, by exendin 9-39. Similarly, the inhibitory effect GLP1 induced on ASC proliferation was reversible in the presence of GI. Since each antagonist has been demonstrated to be selective and specific for its respective receptor (Chepurny et al. 2019), with no cross-activity, these findings suggest that both receptors are necessary for mediating both glucagon and GLP-1 in ASCs, independently of the ligand. This conclusion is further supported by the absence of any synergistic effects in the block of proliferation obtained when glucagon and native GLP-1 were simultaneously administered to ASCs. Both receptor antagonists (GI and $\mathrm{EX}$ ) were also able to revert the inhibitory effect exerted by glucagon on in vitro-induced adipogenesis (assessed by the intracellular lipid droplet quantification and the expression of mature adipocyte markers such as HSL and FABP4), when added during the differentiation. These findings support the existence of some promiscuity between GLP-1R and GCGR. The ability of both the antagonists of each receptor (GI and EX) to prevent glucagon inhibitory activity strongly suggests that glucagon can bind and activate both GCGR and GLP-1R (similarly to what happens in zebrafish and early in the receptor family evolution), and that both receptors are needed for mediating the glucagon activity. The concurrent block of both GLP-1R and GCGR by their respective antagonists reversed the inhibitory effect of glucagon on adipogenesis to a significantly higher extent than the inhibition of the single receptor blockade, suggesting that both receptors contribute to glucagon activity in ASCs. Similarly, both receptors are engaged in response to GLP-1. Interestingly, according to our results, ASCs seemed to express higher levels of GLP-1R compared to GCGR. Alternative mechanism of action of GLP-1 through non-canonical GLP-1R have been proposed to justify the effect of the truncated GLP-1 (9-36) form, which has a very low affinity for the classical receptor (Cantini et al. 2017), including the possibility of heterodimerization with GCGR on the cell surface (Cantini et al. 2016). Further studies are needed to elucidate the nature of the interaction of the two receptors and their reciprocal contribution in mediating the effects of glucagon and GLP-1.

Since the doses of glucagon used in this preclinical, in vitro study, were supraphysiological, the effects observed are not likely to occur in physiological conditions, or even at the increased levels of glucagon typical of obesity and diabetes. However, glucagon concentrations used in our experiments are consistent with the in vitro pharmacological studies.

We still need to improve our knowledge of the mechanisms underlying glucagon and GLP-1 effects on ASCs derived from different adipose depots. In fact, a different activity and susceptibility to metabolic alterations have been described in ASCs from subcutaneous (SAT) and visceral adipose tissue (VAT) (Baglioni et al. 2012). However, the different susceptibility to glucagon and GLP-1 action between different fat depots in obese subjects is not within the aims of this study. Further, specifically designed studies are required for elucidating this aspect.

In conclusion, we demonstrate here for the first time that glucagon can directly act on human ASC interfering with their proliferation and differentiation, in a similar way as other 'proglucagon-derived peptides', such as liraglutide and GLP-1, through a still to be clarified mechanism of action. Glucagon activity requires the engagement of both GCGR and GLP-1R present on ASC surface. This novel glucagon action, here demonstrated in vitro in adipose cells, might contribute to the greater anti-adipose effect of glucagon/GLP-1 agonist combination, or of GLP-1/glucagon dual agonists, in comparison with specific GLP-1 agonists.

\section{Declaration of interest}

G.C., M.T., A.D.F. and M.L. declare no conflict of interest. E.M. has received speaking and/or consulting fees and research grants from AstraZeneca, Boehringer Ingelheim, Eli Lilly, Merck, Novartis, Novo Nordisk, Sanofi, Takeda. 


\section{Funding}

This study was supported by PRIN (2015) (prot. 2015ZTT5KB, Italian Ministry of University and Research) and Ente Cassa di Risparmio di Firenze (prot. 2016/0773)

\section{Acknowledgements}

The authors thank Dr. Tommaso Mello (University of Florence) and Dr. Monica Muratori (University of Florence) for their help in epifluorescence microscopy and cytofluorimetric analysis, respectively.

The authors also acknowledge the bariatric surgeons Dr Marcello Lucchese, Dr Enrico Facchiano and Dr Giovanni Quartararo (Careggi University Hospital, Florence) for the adipose tissue specimens obtained during bariatric surgery from which our group had derived ASC populations stored and used in the present study.

\section{References}

Ahrén B 2015 Glucagon--Early breakthroughs and recent discoveries. Peptides 67 74-81. (https://doi.org/10.1016/j.peptides.2015.03.011)

Arafat AM, Kaczmarek P, Skrzypski M, Pruszyńska-Oszmalek E, Kołodziejski P, Szczepankiewicz D, Sassek M, Wojciechowicz T, Wiedenmann B, Pfeiffer AF, et al. 2013 Glucagon increases circulating fibroblast growth factor 21 independently of endogenous insulin levels: a novel mechanism of glucagon-stimulated lipolysis? Diabetologia 56 588-597. (https://doi.org/10.1007/s00125-0122803-y)

Astrup A \& Finer N 2000 Redefining type 2 diabetes: 'diabesity' or 'obesity dependent diabetes mellitus'? Obesity Reviews 1 57-59. (https://doi.org/10.1046/j.1467-789x.2000.00013.x)

Bagger JI, Holst JJ, Hartmann B, Andersen B, Knop FK \& Vilsbøll T 2015 Effect of oxyntomodulin, glucagon, GLP-1, and combined glucagon +GLP-1 infusion on food intake, appetite, and resting energy expenditure. Journal of Clinical Endocrinology \& Metabolism $1004541-$ 4552. (https://doi.org/10.1210/jc.2015-2335)

Baglioni S, Cantini G, Poli G, Francalanci M, Squecco R, Di Franco A, Borgogni E, Frontera S, Nesi G, Liotta F, et al. 2012 Functional differences in visceral and subcutaneous fat pads originate from differences in the adipose stem cell. PLOS ONE 7 e36569. (https:// doi.org/10.1371/journal.pone.0036569)

Baglioni S, Francalanci M, Squecco R, Lombardi A, Cantini G, Angeli R, Gelmini S, Guasti D, Benvenuti S, Annunziato F, et al. 2009 Characterization of human adult stem-cell populations isolated from visceral and subcutaneous adipose tissue. FASEB Journal $233494-$ 3505. (https://doi.org/10.1096/fj.08-126946)

Bertin E, Arner P, Bolinder J \& Hagström-Toft E 2001 Action of glucagon and glucagon-like peptide-1-(7-36) amide on lipolysis in human subcutaneous adipose tissue and skeletal muscle in vivo. Journal of Clinical Endocrinology \& Metabolism 86 1229-1234. (https://doi. org/10.1210/jcem.86.3.7330)

Cantini G, Di Franco A, Mannucci E \& Luconi M 2017 Is cleaved glucagon-like peptide 1 really inactive? Effects of GLP-1(9-36) on human adipose stem cells. Molecular \& Cellular Endocrinology 439 1015. (https://doi.org/10.1016/j.mce.2016.10.013)

Cantini G, Di Franco A, Samavat J, Forti G, Mannucci E \& Luconi M 2015 Effect of liraglutide on proliferation and differentiation of human adipose stem cells. Molecular \& Cellular Endocrinology 402 4350. (https://doi.org/10.1016/j.mce.2014.12.021)

Cantini G, Mannucci E \& Luconi M 2016 Perspectives in GLP-1 research: new targets, new receptors. Trends in Endocrinology \& Metabolism 27 427-438. (https://doi.org/10.1016/j.tem.2016.03.017)

Chepurny OG, Matsoukas MT, Liapakis G, Leech CA, Milliken BT, Doyle RP \& Holz GG 2019 Non-conventional glucagon and GLP-1 receptor agonist and antagonist interplay at the GLP-1 receptor revealed in high-throughput FRET assays for cAMP. Journal of Biological Chemistry 294 3514-3531. (https://doi.org/10.1074/jbc. RA118.005682)

Day JW, Ottaway N, Patterson JT, Gelfanov V, Smiley D, Gidda J, Findeisen H, Bruemmer D, Drucker DJ, Chaudhary N, et al. 2009 A new glucagon and GLP-1 co-agonist eliminates obesity in rodents. Nature Chemical Biology 5 749-757. (https://doi.org/10.1038/ nchembio.209)

El Bekay R, Coín-Aragüez L, Fernández-García D, Oliva-Olivera W, Bernal-López R, Clemente-Postigo M, Delgado-Lista J, Diaz-Ruiz A, Guzman-Ruiz R, Vázquez-Martínez R, et al. 2016 Effects of glucagonlike peptide- 1 on the differentiation and metabolism of human adipocytes. British Journal of Pharmacology 173 1820-1834. (https:// doi.org/10.1111/bph.13481)

Habegger KM, Heppner KM, Geary N, Bartness TJ, DiMarchi RD \& Tschöp MH 2010 The metabolic actions of glucagon revisited. Nature Reviews. Endocrinology 6 689-697. (https://doi.org/10.1038/ nrendo.2010.187)

Hwang JI, Yun S, Moon MJ, Park CR \& Seong JY 2014 Molecular evolution of GPCRs: GLP1/GLP1 receptors. Journal of Molecular Endocrinology 52 T15-T27. (https://doi.org/10.1530/JME-13-0137)

Irwin DM \& Wong K 2005 Evolution of new hormone function: loss and gain of a receptor. Journal of Heredity 96 205-211. (https://doi. org/10.1093/jhered/esi024)

Lee HM, Joo BS, Lee CH, Kim HY, Ock JH \& Lee YS 2015 Effect of glucagon-like peptide- 1 on the differentiation of adipose-derived stem cells into osteoblasts and adipocytes. Journal of Menopausal Medicine 21 93-103. (https://doi.org/10.6118/jmm.2015.21.2.93)

Monami M, Dicembrini I, Marchionni N, Rotella CM \& Mannucci E 2012 Effects of glucagon-like peptide-1 receptor agonists on body weight: a meta-analysis. Experimental Diabetes Research 2012672658. (https://doi.org/10.1155/2012/672658)

Müller TD, Finan B, Clemmensen C, DiMarchi RD \& Tschöp MH 2017 The new biology and pharmacology of glucagon. Physiological Reviews 97 721-766. (https://doi.org/10.1152/physrev.00025.2016)

Muscogiuri G, Cignarelli A, Giorgino F, Prodam F, Santi D, Tirabassi G, Balercia G, Modica R, Faggiano A \& Colao A 2014 GLP-1: benefits beyond pancreas. Journal of Endocrinological Investigation 371143 1153. (https://doi.org/10.1007/s40618-014-0137-y)

Oren DA, Wei Y, Skrabanek L, Chow BK, Mommsen T \& Mojsov S 2016 Structural mapping and functional characterization of zebrafish class B G-protein coupled receptor (GPCR) with dual ligand selectivity towards GLP-1 and glucagon. PLOS ONE 11 e0167718. (https://doi. org/10.1371/journal.pone.0167718)

Pappachan JM \& Viswanath AK 2017 Medical management of diabesity: do we have realistic targets? Current Diabetes Reports 17 4. (https:// doi.org/10.1007/s11892-017-0828-9)

Pi-Sunyer X, Astrup A, Fujioka K, Greenway F, Halpern A, Krempf M, Lau DC, le Roux CW, Violante Ortiz R, Jensen CB, et al. 2015 A randomized, controlled trial of $3.0 \mathrm{mg}$ of liraglutide in weight management. New England Journal of Medicine 373 11-22. (https:// doi.org/10.1056/NEJMoa1411892)

Pocai A, Carrington PE, Adams JR, Wright M, Eiermann G, Zhu L, Du X, Petrov A, Lassman ME, Jiang G, et al. 2009 Glucagon-like peptide 1/ glucagon receptor dual agonism reverses obesity in mice. Diabetes $\mathbf{5 8}$ 2258-2266. (https://doi.org/10.2337/db09-0278)

Post SR, Rubinstein PG \& Tager HS 1993 Mechanism of action of desHis1-[Glu9]glucagon amide, a peptide antagonist of the glucagon receptor system. Proceedings of the National Academy of Sciences of the United States of America 90 1662-1666. (https://doi.org/10.1073/ pnas.90.5.1662)

Sánchez-Garrido MA, Brandt SJ, Clemmensen C, Müller TD, DiMarchi RD \& Tschöp MH 2017 GLP-1/glucagon receptor co-agonism for treatment of obesity. Diabetologia 60 1851-1861. (https://doi.org/10.1007/s00125-017-4354-8) (c) 2019 Society for Endocrinology Published by Bioscientifica Ltd. Printed in Great Britain 
Sanz C, Vázquez P, Blázquez C, Barrio PA, Alvarez Mdel M \& Blázquez E 2010 Signaling and biological effects of glucagon-like peptide 1 on the differentiation of mesenchymal stem cells from human bone marrow. American Journal of Physiology. Endocrinology \& Metabolism 298 E634-E643. (https://doi.org/10.1152/ajpendo.00460.2009)

Svendsen B, Larsen O, Gabe MBN, Christiansen CB, Rosenkilde MM, Drucker DJ \& Holst JJ 2018 Insulin secretion depends on intra-islet glucagon signaling. Cell Reports 25 1127-1134.e2. (https://doi. org/10.1016/j.celrep.2018.10.018)

Tan TM, Field BC, McCullough KA, Troke RC, Chambers ES, Salem V, Gonzalez Maffe J, Baynes KC, De Silva A, Viardot A, et al. 2013 Coadministration of glucagon-like peptide-1 during glucagon infusion in humans results in increased energy expenditure and amelioration of hyperglycemia. Diabetes 62 1131-1138. (https://doi. org/10.2337/db12-0797)

Thorens B, Porret A, Bühler L, Deng SP, Morel P \& Widmann C 1993 Cloning and functional expression of the human islet GLP-1 receptor. Demonstration that exendin-4 is an agonist and exendin-(9-39) an antagonist of the receptor. Diabetes 421678 1682. (https://doi.org/10.2337/diab.42.11.1678)

Tillner J, Posch MG, Wagner F, Teichert L, Hijazi Y, Einig C, Keil S, Haack T, Wagner M, Bossart M, et al. 2019 A novel dual glucagonlike peptide and glucagon receptor agonist SAR425899: results of randomized, placebo-controlled first-in-human and first-in-patient trials. Diabetes, Obesity \& Metabolism 21 120-128. (https://doi. org/10.1111/dom.13494)

Unson CG, Andreu D, Gurzenda EM \& Merrifield RB 1987 Synthetic peptide antagonists of glucagon. PNAS $\mathbf{8 4} 4083-4087$. (https://doi. org/10.1073/pnas.84.12.4083)

Unson CG, Macdonald D, Ray K, Durrah TL \& Merrifield RB 1991 Position 9 replacement analogs of glucagon uncouple biological activity and receptor binding. Journal of Biological Chemistry 266 2763-2766.

Vendrell J, El Bekay R, Peral B, García-Fuentes E, Megia A, MaciasGonzalez M, Fernández Real J, Jimenez-Gomez Y, Escoté X, Pachón G, et al. 2011 Study of the potential association of adipose tissue GLP-1 receptor with obesity and insulin resistance. Endocrinology 152 4072-4079. (https://doi.org/10.1210/en.2011-1070)

Wewer ANJ, Kuhre RE, Pedersen J, Knop FK \& Holst JJ 2016 The biology of glucagon and the consequences of hyperglucagonemia. Biomarkers in Medicine 10 1141-1151. (https://doi.org/10.2217/bmm-2016-0090)

Wynne K, Park AJ, Small CJ, Patterson M, Ellis SM, Murphy KG, Wren AM, Frost GS, Meeran K, Ghatei MAc et al. 2005 Subcutaneous oxyntomodulin reduces body weight in overweight and obese subjects: a double-blind, randomized, controlled trial. Diabetes 54 2390-2395. (https://doi.org/10.2337/diabetes.54.8.2390)

Received in final form 04 September 2019

Accepted 14 September 2019

Accepted Preprint published online 14 September 2019
(C) 2019 Society for Endocrinology Published by Bioscientifica Ltd. Printed in Great Britain 